\title{
Indoor fungal diversity in primary schools may differently influence allergic sensitization and asthma in children
}

\author{
João Cavaleiro Rufo ${ }^{1,2,3,4}$ (D), Joana Madureira ${ }^{3}$, Inês Paciência ${ }^{1,2,3,4}$, Lívia Aguiar ${ }^{4,5}$, \\ Cristiana Pereira ${ }^{4,5}$, Diana Silva ${ }^{1,2}$, Patrícia Padrão ${ }^{6}$, Pedro Moreira $^{6}{ }^{\prime}$, Luís Delgado ${ }^{1,2}$, \\ Isabella Annesi-Maesano ${ }^{7}$, Eduardo Oliveira Fernandes ${ }^{3}$, João Paulo Teixeira ${ }^{4,5}$ \& \\ André Moreira ${ }^{1,2,4,6}$
}

${ }^{1}$ Basic and Clinical Immunology, Faculty of Medicine, University of Porto, Porto, Portugal; ${ }^{2}$ Centro Hospitalar São João, Porto, Portugal; ${ }^{3}$ INEGI Institute of Science and Innovation in Mechanical Engineering and Industrial Management, Porto, Portugal; ${ }^{4}$ Epidemiology Research Unit, Institute of Public Health (EPIUnit), University of Porto, Porto, Portugal; ${ }^{5}$ National Institute of Health Dr Ricardo Jorge, Porto, Portugal; ${ }^{6}$ Faculty of Nutrition and Food Sciences, University of Porto, Porto, Portugal; ${ }^{7}$ Epidemiology of Allergic and Respiratory Diseases Department, Institut Pierre Louis d'Epidémiologie et de Santé Publique, INSERM and UPC, Paris, France

\begin{abstract}
To cite this article: Cavaleiro Rufo J, Madureira J, Paciência I, Aguiar L, Pereira C, Silva D, Padrão P, Moreira P, Delgado L, Annesi-Maesano I, Oliveira Fernandes E, Teixeira JP, Moreira A. Indoor fungal diversity in primary schools may differently influence allergic sensitization and asthma in children. Pediatr Allergy Immunol 2017: 28 : 332-339.
\end{abstract}

\section{Keywords}

endotoxins; exposure; fungi; indoor air; microbiologic diversity; microbiome; schools; sensitization

\section{Correspondence \\ João Cavaleiro Rufo, Rua Dr. Roberto Frias 4200-465 Porto, Portugal \\ Tel.: +351 225513657 \\ Fax: +351229537352 \\ E-mail: jcrufo@gmail.com}

Accepted for publication 13 February 2017

DOI:10.1111/pai.12704

[Correction added on 31 March 2017 after first online publication: The $6^{\text {th }}$ affiliation was incorrect and has been corrected in this version.]

\begin{abstract}
Background: Childhood exposure to microbiologic agents may influence the development of allergic and respiratory diseases. Apart from home, children spend most of their time at school, which represents an environment of significant exposure to indoor air microbes. Therefore, we aimed to assess how the prevalence of allergic sensitization and asthma in schoolchildren is affected by microbiologic exposure within classrooms.

Methods: Spirometry with bronchodilation, exhaled nitric oxide measurements and skin-prick tests data were retrieved from 858 children aged 8-10 years attending 71 classrooms in 20 primary schools. Air samples were collected in all classrooms using a single-stage microbiologic air impactor through agar plates. Gram-negative endotoxins were collected using flow control pumps and analysed by limulus amebocyte lysate assay. Diversity scores were established as the number of different fungal species found in each classroom.

Results: Classrooms with increased diversity scores showed a significantly lower prevalence of children with atopic sensitization, but not asthma. The risk of sensitization increased with increasing endotoxin exposure in classrooms. Similarly, significantly higher concentrations of Penicillium spp were found in classrooms with a higher number of children with atopic sensitization.

Conclusions: Although no causal relationships could be established, exposure to higher fungal diversity was protective against allergic sensitization but this was not seen for asthma. In contrast, higher exposure to Gram-negative endotoxins and Penicillium spp in primary school's classrooms was associated with increasing odds of allergic sensitization in children.
\end{abstract}

Exposure to indoor air microbiologic agents during childhood has been shown to influence the development of allergic diseases (1). While there is evidence that excessive exposure to indoor air bacteria or fungi concentrations may induce the development of allergy and asthma (2-4), a large number of recent studies also suggest that exposure to certain microorganisms in the early years of life may have a protective effect in children, reducing the risk of allergy and asthma onset by promoting the maturation of Th1 lymphocytes, thus decreasing the incidence of Th2-oriented immunity (hygiene hypothesis) (5-9). There is published evidence supporting the general concept that a low diversity of the human microbiome during infancy is associated with the long-term development of lifestyle-dependent immune disease manifestations, such as atopic disease (10).

Apart from home, young children spend a large section of their daytime at school, mostly within their classrooms, which might be reflected as a long-term exposure to indoor air 
microbes $(5,11)$. If the human microbiome does change according to environmental exposure, the classroom environment might contribute to microbiome changes and, consequently, either induce the development and/or exacerbation of allergy and asthma or, on the other hand, have a protective effect against their onset.

Therefore, the aim of this cross-sectional study was to observe how the prevalence of allergic sensitization and asthma in schoolchildren is influenced by the exposure to indoor air bacteria and fungi in classrooms. More specifically, this work aimed to (i) quantify the concentration of microbiologic parameters in the indoor air of primary school classrooms; and (ii) investigate how microbial concentrations and diversity may influence the risk of asthma and allergy in primary school children.

\section{Methods}

\section{Study design and participants}

A total of 20 public primary schools located in the city of Porto, Portugal, were invited to participate in this crosssectional survey, corresponding to a total of 71 assessed classrooms. The sampling campaigns occurred during the heating season in two different periods: from January to April 2014, and from October 2014 to March 2015 (10 schools per period). The heating season was chosen as the air exchange rates in schools are usually lower during this period. During the sampling, indoor air bacteria, fungi and lipopolysaccharides (LPS) were collected. Concurrently, the clinical assessment of the participating children was performed in the school.

All schools where the study was carried out, along with the Ethical Committee of the University of Porto, approved the study protocol. Children attending the 71 participating classrooms were invited to take part in the study, corresponding to 1602 invited children (Fig. 1). After receiving the written consent from their legal guardians, 916 children were enrolled in the study (participation rate of $57 \%$ ). Considering a confidence level of $95 \%$, the estimated confidence interval for the sample size was 2.42 , showing that the study has a good statistical power. A total of 58 children refused to perform the clinical tests having only the questionnaire data available; thus, spirometry, skin-prick testing and exhaled nitric oxide data were retrieved from 858 children (mean age of $9 \pm 1$ years).

\section{Clinical assessment}

In all participating children, height, weight, lung function (spirometry with bronchodilation) and exhaled level of nitric oxide (NO) were measured and skin-prick tests (SPTs) performed by a trained professional. The parents fulfilled a questionnaire based on the International Study of Asthma and Allergies in Childhood (ISAAC) where they reported the children's symptoms history.

Lung function and airway reversibility were assessed according to the official ATS/ERS guidelines (12). Lung function data were retrieved before and $15 \mathrm{~min}$ after $400 \mu \mathrm{g}$ of inhaled salbutamol.
Airway inflammation was assessed measuring exhaled NO levels using the NObreath (Bedfont Scientific Ltd., Harrietsham, Kent, UK). The results were expressed as parts per billion (ppb) and stratified according to the official ATS guidelines for children (13).

Allergic sensitization was evaluated by SPT on their forearm using a QuickTest ${ }^{\mathrm{TM}}$ (Panatrex Inc., Placentia, California, USA) applicator containing Der p, mix of weeds (Urtica dioica, Plantago lanceolate and Artemisia vulgaris), mix of grasses (Agrostis stolonifera, Anthoxathum odoratum, Dactylis glomerata, Lolium perenne, Arrhenatherium elatius, Festuca rubra, Poa pratensis, Holcus lanatus, Phleum pratense, Secale cereal), cat dander, dog dander and Alt $a$, negative control (extracts dilutant) and a positive control (histamine at $10 \mathrm{mg} / \mathrm{ml}$ ), all belonging to the same batches (Hall Allergy, Netherlands). Results were read 20 min afterwards and allergic sensitization was defined by a positive SPT to at least one of the allergens. If children were on antihistamines or topical corticosteroids on the skin within the previous 7 days, SPTs would be postponed.

The following operational asthma definitions were adopted: (i) clinical criteria - at least a $12 \%$ increase in $\mathrm{FEV}_{1}$ after bronchodilation and over $200 \mathrm{ml}$ and/or asthma diagnosed by a physician with reported symptoms (wheezing, dyspnoea or dry cough) occurring in the past 12 months; (ii) functional criteria at least a $12 \%$ increase in $\mathrm{FEV}_{1}$ after bronchodilation and over $200 \mathrm{ml}$; (iii) treated asthma criteria - asthma diagnosed by a physician and currently under inhaled corticosteroid treatment; and iv) ever asthma - asthma diagnosed by a physician.

Children were classified as having allergic rhinitis when their legal guardians answered positively to the question 'Did the participant suffer from recurrent sneezing, rhinorrhea or nasal congestion in the last 12 months, while not having a cold or flu?'. Atopic dermatitis was defined in accordance with the United Kingdom Working Party's Diagnostic Criteria for Atopic Dermatitis (14). The 'allergic disease' group was comprised by individuals with positive SPT suffering from allergic rhinitis and/or atopic dermatitis.

Characterization of the participants is presented in Table 1. Prevalence of asthma diagnosed by clinical criteria, functional criteria, treated asthma and ever asthma was 9.3\%, 6.6\%, $5.4 \%$ and $6.3 \%$, respectively. Prevalence of allergic sensitization was $34.1 \%$. The prevalence of allergic rhinitis and atopic dermatitis was $10.6 \%$ and $9.3 \%$, respectively.

\section{Microorganism sampling}

Bacterial and fungal air samples were collected using a singlestage microbiologic air impactor (Merck Air Sampler MAS10 0), according to NIOSH method 0800 (15) and EN 13098 (16). Tryptic soy agar (supplemented with $0.25 \%$ cycloheximide) and malt extract agar (supplemented with $1 \%$ of chloramphenicol) were used as culture media for bacteria and fungi, respectively. Air was drawn through the sampler at a $100 \mathrm{l} /$ min rate and sequential duplicate air samples of 2501 were collected. Indoor air samples were obtained from the 71 participating classrooms. A total of two tryptic soy agar and two malt extract agar samples were collected per classroom. The mean CFU values of duplicate samples were used as the final 


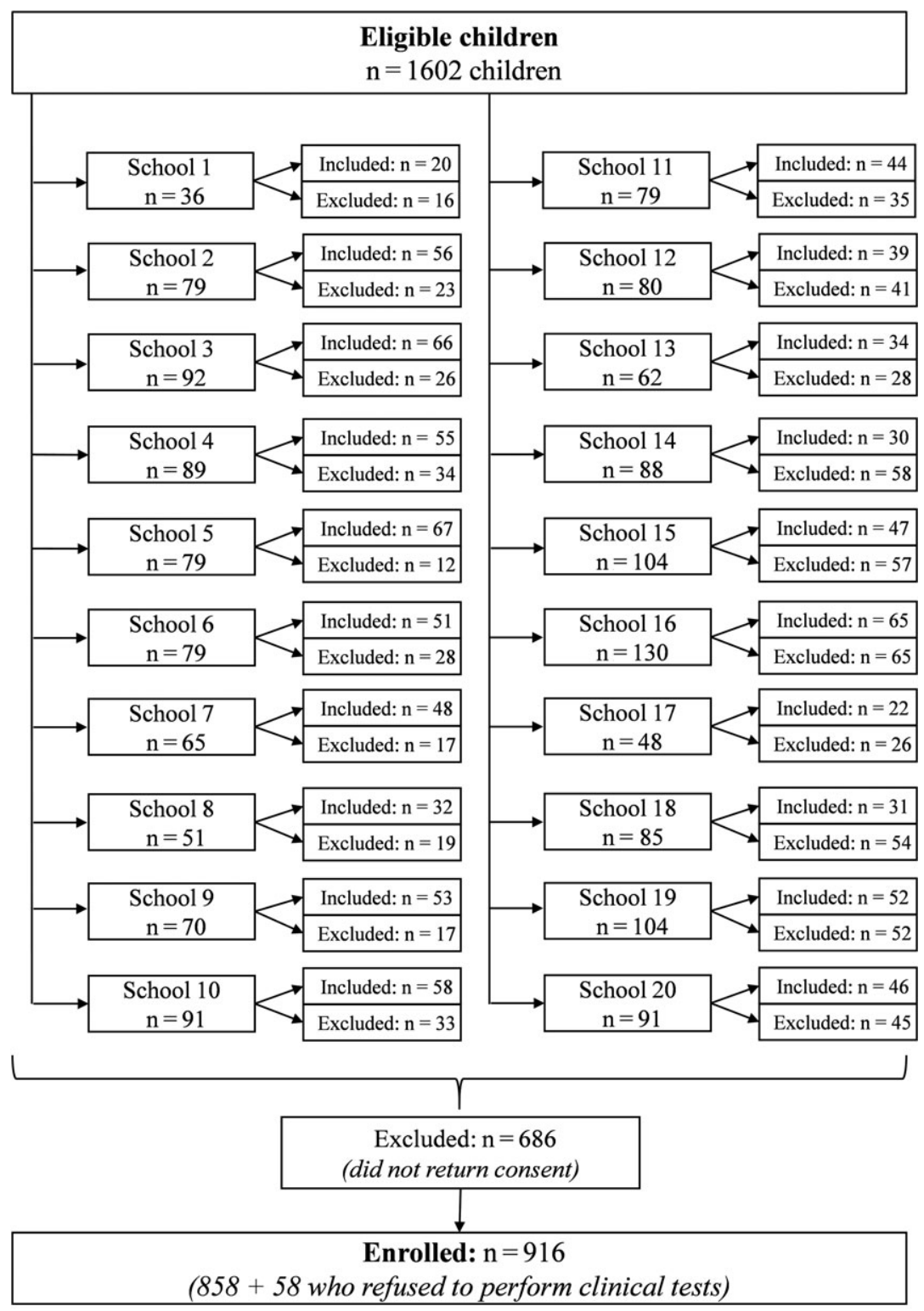

Figure 1 Flow of study participants. result in accordance with the laboratory criteria. The volume, and consequently the duration, of sequential air sampling was the same in all schools and all classrooms. In each sampling day, four field blanks, two sterility blanks, one positive and one negative control per culture medium were used. This methodology has been validated in other works $(17,18)$.

Concurrently with bacteria and fungi assessment, indoor air LPS samples were collected during $4 \mathrm{~h}$ with GilAir-5 flow control pumps (Sensidyne, St. Petersburg, Florida, USA) set to $21 /$ min and coupled to button aerosol stainless steel samplers (SKC Inc., Valley View Road Eighty Four, Pennsylvania, USA).

\section{Laboratory analysis}

Bacterial and fungal samples were incubated at $37 \pm 1^{\circ} \mathrm{C}$ for $48 \pm 3 \mathrm{~h}$ and at $25 \pm 3^{\circ} \mathrm{C}$ for $72 \pm 3 \mathrm{~h}$, respectively (16-18).
Quantification of bacteria and fungi levels was performed by naked eye count following an internal procedure based on the methodologies expressed in EN 13098 (16) and ISO 4833-1:2013 (19). The number of colonies recovered on the air sample plates was adjusted using a positive hole correction factor, and the results were expressed as number of colonyforming units per cubic metre of air $(\mathrm{CFU} / \mathrm{m} 3)$. The correction factor was based on Fellers law. The quantification limit was established as $10 \mathrm{CFU}$ per plate.

Specific fungal identification was performed 7 days after incubation, either on the original sampling media-MEA plates or after subculturing procedures, whenever colony isolation and growth observation were needed. Identification of fungal colonies was based upon phenotypic characteristics and followed standard mycological procedures based on their micro and macro-morphological characteristics. 
Table 1 Characteristics of the participants

\begin{tabular}{|c|c|c|c|}
\hline & $\begin{array}{l}\text { Total } \\
\mathrm{n}=858\end{array}$ & $\begin{array}{l}\text { Female } \\
n=427\end{array}$ & $\begin{array}{l}\text { Male } \\
n=431\end{array}$ \\
\hline Age (years, mean \pm sd) & $9 \pm 1$ & $9 \pm 1$ & $9 \pm 1$ \\
\hline Weight $(\mathrm{kg})$ & $31.2(27.3-37.2)$ & $31.8(27.0-37.3)$ & $30.8(27.5-36.8)$ \\
\hline Height (cm) & $135(130-140)$ & 135 (130-139) & 135 (130-140) \\
\hline BMI $\left(\mathrm{kg} / \mathrm{m}^{2}\right)$ & $17.0(15.5-19.5)$ & $17.3(15.6-19.8)$ & $16.8(15.5-19.2)$ \\
\hline \multicolumn{4}{|l|}{ Clinical } \\
\hline Allergic sensitization (n) & 293 & 139 & 154 \\
\hline Asthma (n) & 73 & 43 & 30 \\
\hline Allergic rhinitis (n) & 91 & 37 & 54 \\
\hline Atopic dermatitis (n) & 80 & 37 & 43 \\
\hline Allergic disease $(n)^{*}$ & 101 & 40 & 61 \\
\hline \multicolumn{4}{|l|}{ Lung function } \\
\hline $\mathrm{FEV}_{1}(\mathrm{I})$ & $1.75(1.58-1.95)$ & $1.71(1.55-1.92)$ & 1.77 (1.59-1.99) \\
\hline FVC (I) & $1.89(1.69-2.14)$ & $1.83(1.66-2.08)$ & $1.94(1.72-2.18)$ \\
\hline $\mathrm{FEF}_{25-75}(\mathrm{I} / \mathrm{s})$ & $2.28(1.92-2.65)$ & $2.28(1.93-2.64)$ & $2.29(1.91-2.68)$ \\
\hline FEV1/FVC (\%) & $92.7(88.9-96.4)$ & $93.2(89.8-96.6)$ & $92.0(88.0-96.1)$ \\
\hline FEV 1 reversibility (\%) & $3.5(0.0-7.1)$ & $3.7(0.0-7.2)$ & $3.5(0.5-7.0)$ \\
\hline FEV ${ }_{1}$ reversibility $(\mathrm{ml})$ & $60(0-120)$ & $60(0-120)$ & $60(10-130)$ \\
\hline Exhaled NO (ppb) & $11.0(6.0-20.0)$ & $9.5(5.0-16.0)$ & $12.0(6.0-22.5)$ \\
\hline
\end{tabular}

$\mathrm{BMI}$, body mass index; $\mathrm{FEV}_{1}$, forced expiratory volume in the first second of FVC; FVC, forced vital capacity; FEF $25-75$, forced expiratory flow middle portion of FVC.

*The "allergic disease" group was comprised by individuals with positive SPT suffering from allergic rhinitis and/or atopic dermatitis.

Data reported as median (25-75\%) unless otherwise stated.

For endotoxin extraction, sample filters were eluted in $5 \mathrm{ml}$ extraction solution (Pyrogen Free Water plus $0.05 \%$ Tween20) and rocked vigorously for $1 \mathrm{~h}$ at room temperature on a horizontal shaker. After $10 \mathrm{~min}$ of centrifugation at $1000 \mathrm{~g}$, total supernatant per sample was collected and analysed. Endotoxin quantification was performed using the limulus amebocyte lysate (LAL) Kinetic-QCLTM (Lonza ${ }^{\circledR}$, Pontevedra, Spain) following the manufacturer's guidelines. Endotoxin concentrations were expressed as $\mathrm{EU} / \mathrm{m}^{3}$. The limit of detection for the LAL Kinetic-QCLTM is $0.005 \mathrm{EU} / \mathrm{ml}$, corresponding to $0.025 \mathrm{EU} / \mathrm{m}^{3}$ under the adopted procedure.

\section{Statistical analysis}

The SPSS ${ }^{\circledR}$ statistical package software v20.0 (IBM, USA) was used to statistically analyse the data. The Kolmogorov-Smirnov test was used to check continuous variables for normality. As non-Gaussians distributions were observed, the MannWhitney test was used for inferential analysis.

When analysing the specific fungi species, each species' proportions were generally categorized into tertiles. As in some cases the detected levels were so low, such as Acremonium spp., the variable was dichotomized with the lower category consisting in values under the limit of detection and the higher category including those over the detection limit.

Microbial diversity score was defined as the sum of all the detected fungi groups/species. Due to the low number of observations with low and high diversity, the variable was categorized into quartiles, being the classrooms with lower diversity score in the first quartile, whereas the fourth quartile included those with higher diversity scores. Distributions of parameters by classroom, as well as diversity scores, are presented in Table S1.

Logistic regression adjusted for age and height was used to analyse the risk for allergic sensitization and asthma associated with the collected indoor air microbiology (total and specific values). Multinomial logistic regression was used for analysing the risk of inflammation reported by exhaled NO. The results were expressed as odds ratio (OR) and respective 95\% confidence interval $(95 \% \mathrm{CI})$.

\section{Results}

Classrooms with second tertile $(\mathrm{OR}=1.26$ [95\% CI: 0.88 1.79]) and third tertile ( $\mathrm{OR}=1.40$ [95\% CI: 0.99-1.99]) concentrations of LPS showed a tendency for a higher risk of allergic sensitization (Fig. 2a). To further explore the possibility of LPS concentrations in classrooms being associated with the prevalence of allergic sensitization, nonparametric tests for independent samples were performed (Mann-Whitney test). The results showed that classrooms with higher concentrations of LPS had a significantly higher prevalence of allergic children (2.20 [0.89-4.25] vs. 2.60 [1.18-5.68], data presented as median [25-75\%], respectively, non-sensitized vs sensitized, $\mathrm{p}=0.02$ ).

Diversity scores ranged from 1 to 11 . Logistic regression models showed that there is a negative association between the number of fungi species in classrooms and the risk of allergic sensitization (Fig. 2b). These results were significant for the third (0.63 [95\% CI: $0.40-0.98])$ and fourth (0.60 [95\% CI: 0.40 0.92]) quartile scores, which ranged from 7 to 8 (Table S1).

Additional pro-sensitization evidence was observed with specific fungal species, as classrooms with second tertile 
(a)

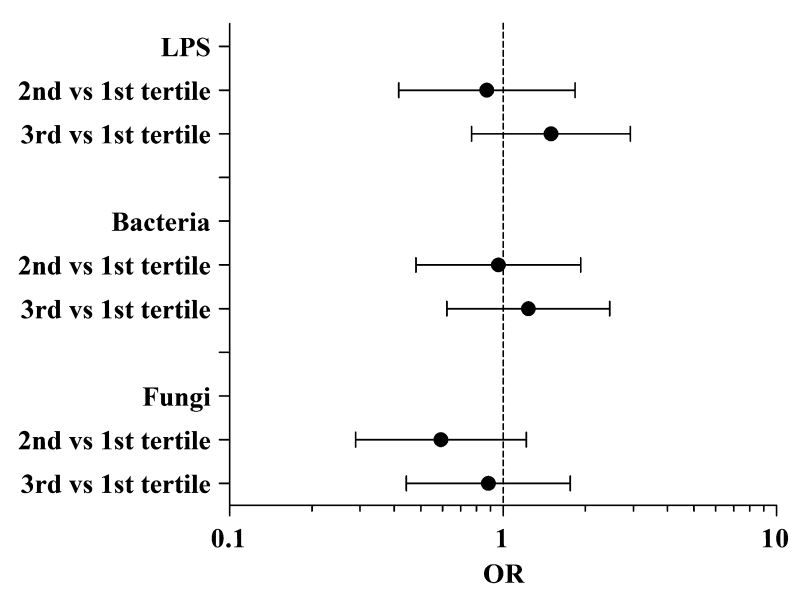

(b)

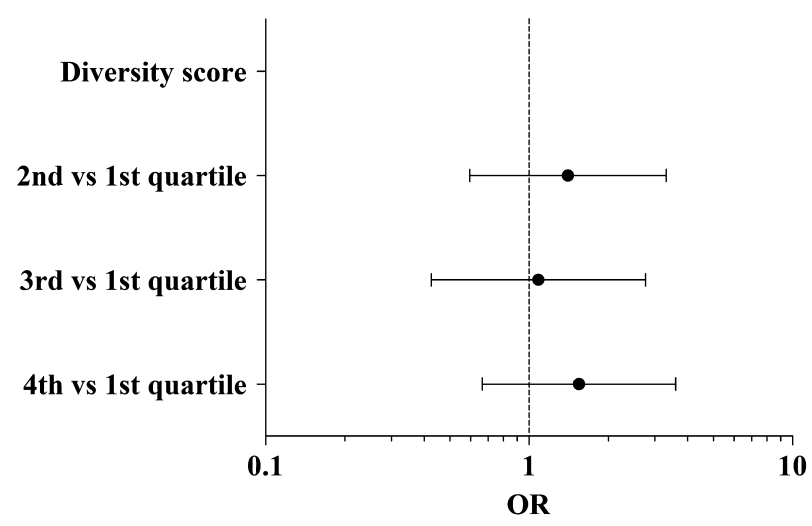

\section{Allergic sensitization}

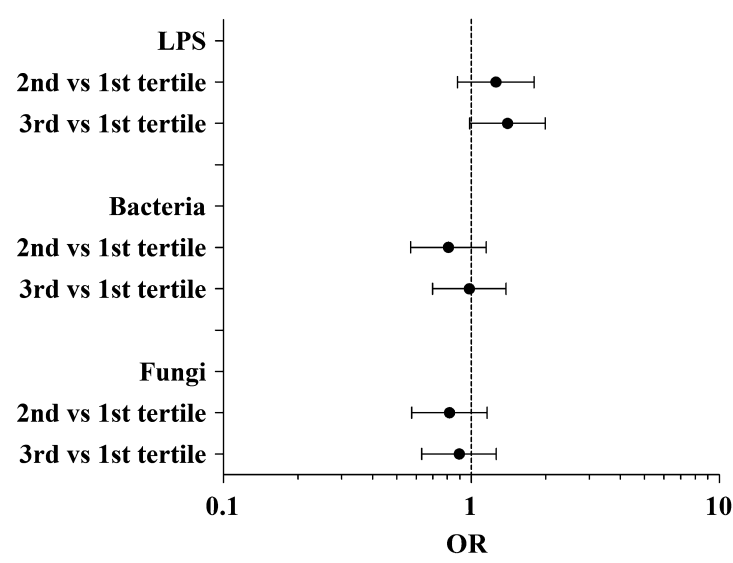

Allergic sensitization

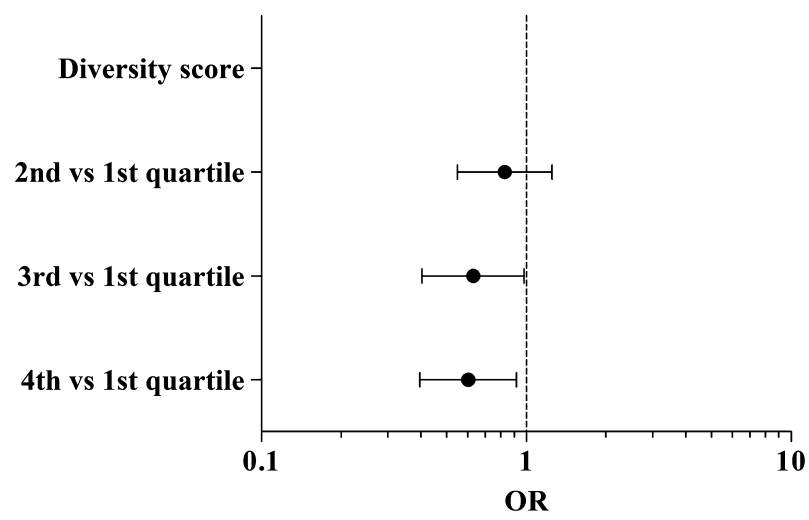

Figure 2 (a) Logistic regression between LPS, bacterial and fungal exposure in classrooms and the prevalence of asthma and atopy. (b) Logistic regression between fungal diversity scores and the prevalence of asthma and atopy. The results are expressed as odds ratios with $95 \% \mathrm{Cl}$ in logarithmic scale.

concentrations of Penicillium spp were associated with a higher risk of sensitization $(\mathrm{OR}=1.46[95 \% \mathrm{CI}$ : 1.02-2.09]) and classrooms with third tertile concentrations showed an even higher risk $(\mathrm{OR}=1.68$ [95\% CI: $1.18-2.40])$. This tendency for a higher sensitization risk associated with Penicillium spp can be observed in Fig. 3. On the other hand, classrooms with higher concentrations of Aspergillus fumigatus (third vs first tertile, $\mathrm{OR}=0.64 \quad[95 \%$ CI: 0.47-0.87]), Aspergillus niger (detected vs not detected, OR $=0.62$ [95\% CI: $0.45-0.87]$ ), Chaetomium spp (detected vs not detected, OR $=0.61$ [95\% CI: 0.39-0.96]) and Rhizopus spp (detected vs not detected, $\mathrm{OR}=0.62$ [95\% CI: 0.45-0.87]) were associated with a lower risk of allergic sensitization (Fig. 3). Interestingly, the risk ratios were very similar between these species (children had 1.56-1.64 times less risk of being allergic).

To further explore the association of fungal diversity with the atopic syndrome, the risk of individuals with positive SPT suffering from allergic rhinitis and/or atopic dermatitis was also investigated. The results showed no significant differences between the quartiles of fungal diversity (Fig. S4).
No associations between LPS, bacteria or fungi concentrations in classrooms were found for any of the four definitions of asthma (Fig. S1). Also, no associations were observed between LPS, bacteria and fungi concentrations and the risk for having low or high exhaled NO (Fig. S2). The classrooms' specific fungal species and flora diversity were also not associated as a risk factor for any of the four different definitions of asthma (Fig. S3). Similar results were observed for exhaled NO values (data not shown).

\section{Discussion}

Our findings suggest that higher levels of endotoxin or Penicillium exposure in classrooms is associated with increased risk of allergic sensitization in school-aged children, while a higher fungal diversity showed a clear tendency for a decreased sensitization. However, similar tendencies were not observed when considering asthma or exhaled NO.

The main limitation of our study resides in its nature, which does not allow causal relationships to be established. 

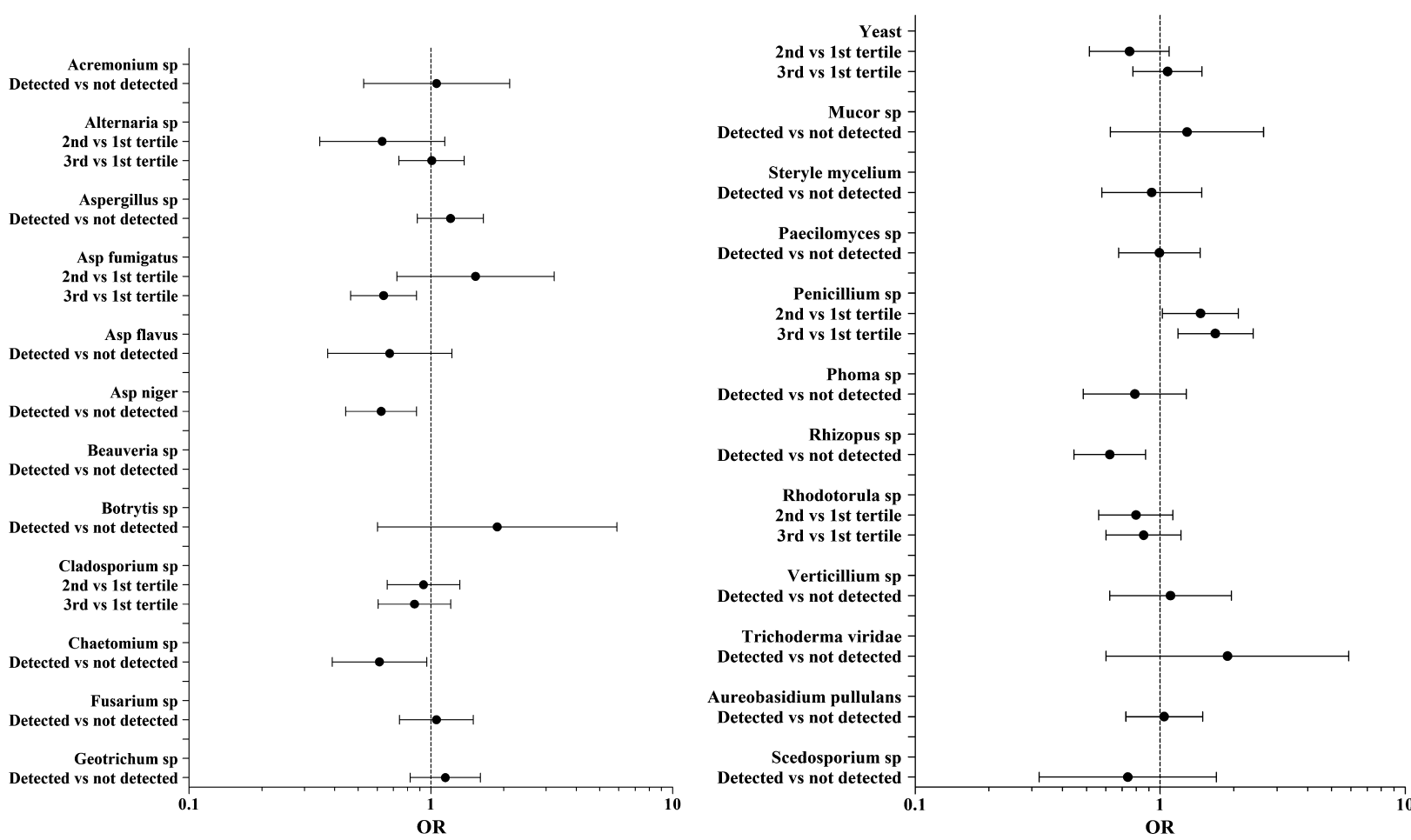

Figure 3 Logistic regression between exposure to specific fungi species in classrooms and the prevalence of atopy. The results are expressed as odds ratios with $95 \% \mathrm{Cl}$ in logarithmic scale.

Moreover, our observations consider only school-time exposure. While children spend most of their day at school, inside the classroom, they are still under exposure to microbiologic agents in other environments when they sleep, or on the playground, during recess; therefore, the complete exposome should be considered in future studies. Also, although in Portugal children usually stay in the same classroom through primary school, this may not be true in other countries, resulting in more exposure environments. Additionally, sensitization to one or more allergens does not necessarily imply a clinically relevant allergic status. Nevertheless, it was still possible to observe tendencies on how the prevalence of allergy and asthma in schoolchildren was affected by the exposure to indoor air bacteria and fungi in classrooms.

The strengths of this work reside in the substantial number of participants, either concerning classrooms or clinically analysed children. Moreover, the four distinct definitions of asthma prevent bias associated with the inclusion or exclusion of asymptomatic individuals and the diversity scores in the present study were calculated based on a large set of fungal species (24 in total), which enhances the impact of the results.

Endotoxin concentrations in classrooms were associated with a higher prevalence of sensitization to inhalant allergens in children, somehow corroborating the results obtained in the PASTURE study (20). These results need to be interpreted with caution, however, as children in the present study were considerably older and may have their immune systems further developed; thus, the impact of exposure to LPS in this age group may be substantially different than when exposition occurs in pre- and post-natal periods of life. This may also justify why, unlike in previous studies $(5,7,9,20,21)$, exposure to environmental LPS did not show any significant association with asthma prevalence. A further matured immune system may also be responsible for the distinct results regarding exhaled NO associations with microbiologic parameters between the present work and the study performed by Casas et al. (22), which showed that exhaled NO at school age was lower in children exposed to LPS during the first 2-3 months of life.

Although no significant associations between exposure to total bacteria concentrations and allergic sensitization, asthma or exhaled nitric oxide levels were found, total fungi concentration is generally not a good predictor as it is not very specific and does not consider several confounding factors such as the species and diversity involved $(1,23)$. In this study, no fungi species in classrooms were associated with a lower prevalence of the four definitions of asthma. In contrast, the risk for sensitization was significantly lower in classrooms with higher concentrations of some Aspergillus species (along with Chaetomium and Rhizopus species), thus supporting the results reported by Ege et al. (5) within the GABRIELA study.

Classrooms with higher concentrations of Penicillium spp showed a significantly higher prevalence of allergic sensitization. This result corroborates those obtained by Sharpe et al. (24), which showed that Penicillium species were found in significantly higher concentrations in homes of individuals with 
asthma. It is possible that this result is associated with the lower diversity scores in the respective classrooms, as the high concentrations of Penicillium spp may extensively reduce the sustainability of other fungi species. In contrast, classrooms with a higher fungal diversity and with lower concentrations of Penicillium spp were shown to be negatively associated with the prevalence of allergic sensitization, supporting the results from previous studies $(25,26)$. Despite having a clear impact on sensitization, fungal diversity was not a risk factor for allergic rhinitis and atopic dermatitis, suggesting that exposure to microbiologic agents may differently influence the atopic syndrome.

A different risk pattern for fungal diversity between the different definitions of asthma was also discernable, with the fungal diversity showing a tendency to be associated with a higher risk for lung function diagnosed asthma, but not for the other definitions. Sensitization odds ratio was lower than the unity for all tertiles, and a significant tendency for a lower risk of allergic sensitization was observed in classrooms with higher fungal diversity. This suggests that exposure to environmental fungi may have a completely different impact in asthma and allergy.

Part of these results are in line with recent advances in the biodiversity hypothesis (27), supporting the importance of exposure to a diverse biome to prevent development of allergic sensitization. Similarly to the results found by Ruokolainen et al. (28) in green areas around cities, microbiota biodiversity in classrooms seems to have an important role in the early immunomodulation. On the other hand, this study's results failed to support the evidence of beneficial exposure to bacterial endotoxins as observed in other studies concerning the biodiversity hypothesis (29). However, these are not isolated results. For instance, a domestic endotoxin exposure study in Cyprus also found higher exposure to endotoxins to be associated with increased allergic sensitization (30). It is possible that regional environmental and genotypic differences (for instance, Mediterranean versus Nordic regions) may have a role in this controversy. More region-wide studies are needed to further clarify the biodiversity hypothesis.

In conclusion, the microbiologic diversity in classrooms is associated with a lower risk of sensitization development in children, thus supporting the results from other studies concerning home exposure. However, this was not observed for the prevalence of asthma, suggesting that exposure to microbiologic agents may have a different mechanism of impact in both diseases. The four different asthma definitions used in this study further support this result. In face of these evidences, understanding the factors that influence diversity in the school environment may lead to public health recommendations for reducing the development of allergic sensitization or prevent symptomatic exacerbations in the future.

\section{Acknowledgments}

Authors gratefully acknowledge the funding by Fundação para a Ciência e Tecnologia through the ARIA project (PTDC/DTP-SAP/1522/2012), through the scholarships SFRH/BD/108605/2015 and SFRH/BD/112269/ 2015 and by the Project NORTE-01-0145-FEDER-000010 - Health, Comfort and Energy in the Built Environment (HEBE), cofinanced by Programa Operacional Regional do Norte (NORTE2020), through Fundo Europeu de Desenvolvimento Regional (FEDER). The authors are thankful to Mariana Pinto for her participation in the clinical assessment.

\section{References}

1. Karvonen AM, Hyvarinen A, Rintala H, et al. Quantity and diversity of environmental microbial exposure and development of asthma: a birth cohort study. Allergy 2014: 69: 1092-101.

2. Bush RK, Portnoy JM, Saxon A, Terr AI, Wood RA. The medical effects of mold exposure. J Allergy Clin Immunol 2006: 117: 326-33.

3. Mendell MJ, Mirer AG, Cheung K, Tong M, Douwes J. Respiratory and allergic health effects of dampness, mold, and dampness-related agents: a review of the epidemiologic evidence. Environ Health Perspect 2011: 119: 748-56.

4. Bornehag CG, Sundell J, Hagerhed-Engman L, Sigsggard T, Janson S, Aberg N. 'Dampness' at home and its association with airway, nose, and skin symptoms among 10,851 preschool children in Sweden: a cross-sectional study. Indoor Air 2005: 10: 48-55.

5. Ege MJ, Mayer M, Normand A-C, et al. Exposure to environmental microorganisms and childhood asthma. $N$ Engl J Med 2011: 364: 701-9.
6. Gehring U, Heinrich J, Hoek G, et al. Bacteria and mould components in house dust and children's allergic sensitisation. Eur Respir J 2007: 29: 1144-53.

7. Mendy A, Gasana J, Vieira ER, et al. Endotoxin exposure and childhood wheeze and asthma: a meta-analysis of observational studies. J Asthma 2011: 48: 685-93.

8. Schram-Bijkerk D, Doekes G, Douwes J, et al. Bacterial and fungal agents in house dust and wheeze in children: the PARSIFAL study. Clin Exp Allergy 2005: 35: 1272-8.

9. Braun-Fahrlander C, Riedler J, Herz U, et al. Environmental exposure to endotoxin and its relation to asthma in school-age children. $N$ Engl J Med 2002: 347: 869-77.

10. Bisgaard H, Li N, Bonnelykke K, et al. Reduced diversity of the intestinal microbiota during infancy is associated with increased risk of allergic disease at school age. J Allergy Clin Immunol 2011: 128: 646-52.

11. Morawska L, Afshari A, Bae GN, et al. Indoor aerosols: from personal exposure to risk assessment. Indoor Air 2013: 23: 462-87.
12. Miller MR, Hankinson J, Brusasco V, et al. Standardisation of spirometry. Eur Respir $J$ 2005: 26: 319-38.

13. Dweik RA, Boggs PB, Erzurum SC, et al. An official ATS clinical practice guideline: interpretation of exhaled nitric oxide levels (FENO) for clinical applications. Am J Respir Crit Care Med 2011: 184: 602-15.

14. Williams HC, Burney PG, Pembroke AC, Hay RJ. The U.K. working party's diagnostic criteria for atopic dermatitis. III. Independent hospital validation. $\mathrm{Br} J$ Dermatol 1994: 131: 406-16.

15. National Institute for Occupational Safety and Health. NIOSH manual of analytical methods 1998.

16. Standard E. CSN EN 13098: workplace atmosphere - guidelines for measurement of airborne micro-organisms and endotoxin. 2000.

17. Madureira J, Paciência I, Rufo JC, Pereira C, Teixeira JP, de Oliveira Fernandes E. Assessment and determinants of airborne bacterial and fungal concentrations in different indoor environments: Homes, child day-care centres, primary schools and 
elderly care centres. Atmos Environ 2015 : 109: 139-46.

18. Madureira J, Pereira C, Paciência I, Teixeira JP, de Oliveira Fernandes E. Identification and levels of airborne fungi in Portuguese primary schools. $J$ Toxicol Environ Health $A$ 2014: 77: 816-26.

19. International Organization for Standardization. 4833-1: microbiology of the food chain - horizontal method for the enumeration of microorganisms - part 1 : colony count at 30 degrees $\mathrm{C}$ by the pour plate technique. 2013.

20. Karvonen AM, Hyvarinen A, Gehring U, et al. Exposure to microbial agents in house dust and wheezing, atopic dermatitis and atopic sensitization in early childhood: a birth cohort study in rural areas. Clin Exp Allergy 2012: 42: 1246-56.

21. Thorne PS, Kulhankova K, Yin M, Cohn R, Arbes SJ Jr, Zeldin DC. Endotoxin exposure is a risk factor for asthma: the national survey of endotoxin in United
States housing. Am J Respir Crit Care Med 2005: 172: 1371-7.

22. Casas L, Tischer C, Wouters IM, et al. Early life microbial exposure and fractional exhaled nitric oxide in school-age children: a prospective birth cohort study. Environ Health 2013: 12: 12-103.

23. Newson R, Strachan D, Corden J, Millington W. Fungal and other spore counts as predictors of admissions for asthma in the Trent region. Occup Environ Med 2000: 57: 786-92.

24. Sharpe RA, Bearman N, Thornton CR, Husk K, Osborne NJ. Indoor fungal diversity and asthma: a meta-analysis and systematic review of risk factors. $J$ Allergy Clin Immunol 2015: 135: 110-22.

25. Behbod B, Sordillo JE, Hoffman EB, et al. Asthma and allergy development: contrasting influences of yeasts and other fungal exposures. Clin Exp Allergy 2015: 45: 154-63.
26. Dannemiller KC, Mendell MJ, Macher JM, et al. Next-generation DNA sequencing reveals that low fungal diversity in house dust is associated with childhood asthma development. Indoor Air 2014: 24: 236-47.

27. Haahtela T. What is needed for allergic children? Pediatr Allergy Immunol 2014: 25: 21-4.

28. Ruokolainen L, von Hertzen L, Fyhrquist $\mathrm{N}$, et al. Green areas around homes reduce atopic sensitization in children. Allergy 2015: 70: 195-202.

29. Carlsten C, Ferguson A, Dimich-Ward H, et al. Association between endotoxin and mite allergen exposure with asthma and specific sensitization at age 7 in high-risk children. Pediatr Allergy Immunol 2011: 22: 320-6.

30. Nicolaou N, Yiallouros P, Pipis S, Ioannou P, Simpson A, Custovic A. Domestic allergen and endotoxin exposure and allergic sensitization in Cyprus. Pediatr Allergy Immunol 2006: 17: 17-21.

\section{Supporting Information}

Additional Supporting Information may be found in the online version of this article:

Figure S1. Logistic regression between LPS, bacterial and fungal exposure in classrooms and the prevalence of asthma (considering the four different classification methods). The results are expressed as odds ratios with $95 \%$ CI in logarithmic scale.

Figure S2. Logistic regression between LPS, bacterial and fungal exposure in classrooms and the levels of FeNO. The results are expressed as odds ratios with $95 \% \mathrm{CI}$ in logarithmic scale.
Figure S3. Logistic regression between fungal diversity scores in classrooms and the prevalence of asthma (considering the four different classification methods). The results are expressed as odds ratios with $95 \% \mathrm{CI}$ in logarithmic scale.

Figure S4. Logistic regression between fungal diversity scores in classrooms and the prevalence of allergic disease (positive skin-prick tests with reported allergic rhinitis and/or atopic dermatitis). The results are expressed as odds ratios with $95 \% \mathrm{CI}$ in logarithmic scale.

Table S1. Concentration of microbial agents and distribution of fungal species measured in the participating classrooms. 